\title{
Prediction of microvascular invasion of hepatocellular carcinoma: value of volumetric iodine quantification using preoperative dual-energy computed tomography
}

Taek Min Kim², Jeong Min Lee ${ }^{1,2,3^{*}}$ (D) Jeong Hee Yoon ${ }^{1,2}$, ljin Joo ${ }^{1,2}$, Sae-Jin Park ${ }^{1,2}$, Sun Kyung Jeon ${ }^{1,2}$, Bernhard Schmidt ${ }^{4}$ and Sedlmair Martin ${ }^{4}$

\begin{abstract}
Background: To investigate the potential value of volumetric iodine quantification using preoperative dual-energy computed tomography (DECT) for predicting microvascular invasion (MVI) of hepatocellular carcinoma (HCC).

Methods: This retrospective study included patients with single HCC treated through surgical resection who underwent preoperative DECT. Quantitative DECT features, including normalized iodine concentration (NIC) to the aorta and mixed-energy $C T$ attenuation value in the arterial phase, were three-dimensionally measured for peritumoral and intratumoral regions: (i) layer-by-layer analysis for peritumoral layers (outer layers 1 and 2; numbered in close order from the tumor boundary) and intratumoral layers (inner layers 1 and 2) with 2-mm layer thickness and (ii) volume of interest (VOI)-based analysis with different volume coverage (tumor itself; $\mathrm{VOI}_{\mathrm{O}}$, tumor plus outer layer 1 ; $\mathrm{VOl}_{\mathrm{O} 2}$, tumor plus outer layers 1 and 2; $\mathrm{VOI}_{11}$, tumor minus inner layer 1 ; $\mathrm{VOI}_{12}$, tumor minus inner layers 1 and 2). In addition, qualitative CT features, including peritumoral enhancement and tumor margin, were assessed. Qualitative and quantitative CT features were compared between HCC patients with and without MVI. Diagnostic performance of DECT parameters of layers and VOls was assessed using receiver operating characteristic curve analysis.

Results: A total of 36 patients (24 men, mean age $59.9 \pm 8.5$ years) with MVI $(n=14)$ and without MVI $(n=22)$ were included. HCCS with MVI showed significantly higher NICs of outer layer 1, outer layer 2, $\mathrm{VOl}_{\mathrm{O} 1}$, and $\mathrm{VOl}_{\mathrm{O} 2}$ than those without MVI $(P=0.01,0.04,0.02,0.02$, respectively). Among the NICs of layers and VOls, the highest area under the curve was obtained in outer layer 1 (0.747). Qualitative features, including peritumoral enhancement and tumor margin, and the mean $C T$ attenuation of each layer and each VOI were not significantly different between HCCs with and without MVI (both $P>0.05)$.

(Continued on next page)
\end{abstract}

\footnotetext{
* Correspondence: jmlshy2000@gmail.com

'Department of Radiology, Seoul National University Hospital, Seoul, South Korea

${ }^{2}$ Department of Radiology, Seoul National University College of Medicine, Seoul, South Korea

Full list of author information is available at the end of the article
}

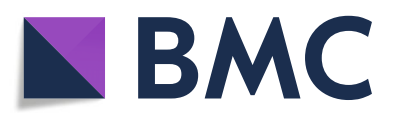

(- The Author(s). 2020 Open Access This article is licensed under a Creative Commons Attribution 4.0 International License, which permits use, sharing, adaptation, distribution and reproduction in any medium or format, as long as you give appropriate credit to the original author(s) and the source, provide a link to the Creative Commons licence, and indicate if changes were made. The images or other third party material in this article are included in the article's Creative Commons licence, unless indicated otherwise in a credit line to the material. If material is not included in the article's Creative Commons licence and your intended use is not permitted by statutory regulation or exceeds the permitted use, you will need to obtain permission directly from the copyright holder. To view a copy of this licence, visit http://creativecommons.org/licenses/by/4.0/. The Creative Commons Public Domain Dedication waiver (http://creativecommons.org/publicdomain/zero/1.0/) applies to the data made available in this article, unless otherwise stated in a credit line to the data. 
(Continued from previous page)

Conclusions: Volumetric iodine quantification of peritumoral and intratumoral regions in arterial phase using DECT may help predict the MVI of HCC.

Keywords: Hepatocellular carcinoma, Microvascular invasion, Dual-energy CT, Peritumoral enhancement, lodine quantification, lodine concentration

\section{Background}

Hepatocellular carcinoma (HCC) is the most common primary liver cancer and is the second leading cause of cancer-related deaths in the Asia-Pacific region [1]. Liver resection and liver transplantation are the first-line curative treatments for eligible patients, but recurrence after surgical treatment is frequent. Previous studies have reported a 5-year recurrence rate of $25 \%$ after liver transplantation and $70 \%$ after liver resection [2, 3]. Vascular invasion is a prognostic factor for predicting recurrence and overall survival [4]. In particular, microvascular invasion (MVI) is known to be responsible for early recurrence within the first 2 years after curative treatments [5]. Thus, we may be able to identify patients at risk of developing early recurrence if we can preoperatively diagnose MVI.

Previous studies reported that several imaging features of the tumor border on preoperative imaging were associated with MVI of HCC. These features include non-smooth tumor margins, peritumoral enhancement in the arterial phase, peritumoral hypointensity in the hepatobiliary phase, and radiogenomic algorithm based on the association between imaging features (internal arteries and hypoattenuating halos) [6-10]. This is reasonable because, in general, a peripheral portion of many malignant tumors may have the most proliferative or aggressive biological behavior for invading adjacent parenchyma. However, preoperative MVI diagnosis is challenging, and the imaging findings and definition of "peri-" tumoral tissue are subjective and highly readerdependent. Therefore, if we can reduce the variation and quantify the enhancement of peritumoral tissue, it would be helpful for improving the diagnostic performance of the arterial phase to identify MVI in HCC. Given that material decomposition and iodine quantification by dual-energy CT have been used for many oncologic applications, such as differentiating tumor types and assessing the treatment response of the tumor [11-14], we surmised that iodine concentration could be used for the quantitative assessment of peritumoral enhancement of HCC.

Therefore, the purpose of this study was to identify the potential diagnostic value of iodine quantification in the peritumoral region of $\mathrm{HCC}$ by volumetric tumor segmentation with dual-energy computed tomography (DECT) for identifying MVI.

\section{Methods}

The institutional review board of our institution approved this retrospective study and waived the requirement for informed consent.

\section{Study population}

We retrospectively searched our radiology database system between April 2017 and December 2019 to identify eligible patients according to the following criteria: (a) underwent four-phase liver $\mathrm{CT}$ at dual-source dualenergy CT (SOMATOM Force; Siemens Healthineers, Forchheim, Germany); (b) underwent hepatic resection within 4 weeks after CT; and (c) surgically confirmed single, treatment-naïve HCC. The exclusion criteria were as follows: (a) preoperative local treatment for the index tumor; (b) $<1 \mathrm{~cm}$, which may increase registration error; and (c) definite macrovascular invasion.

\section{Histopathology}

All surgical specimens were examined by experienced pathologists. The histologic parameters included size, number, Edmondson-Steiner grade, and MVI status of the resected tumor. MVI was defined as the presence of tumor cells in the portal vein, hepatic vein, or a large capsular vessel of the surrounding hepatic tissue lined by the endothelium that was visible only on microscopy. Additionally, the histological grade of fibrosis in the background liver parenchyma was reported on the basis of the staging system for chronic hepatitis by the Korean Study Group for the Pathology of Digestive Diseases (0, no fibrosis; 1, portal fibrosis; 2, periportal fibrosis; 3 , septal fibrosis; 4, cirrhosis) [15].

\section{CT protocol}

Liver CT consisted of precontrast, arterial, portal venous, and equilibrium phases. Precontrast, portal venous, and equilibrium phase images were obtained using 90$\mathrm{kVp}$ tube energy before, $70 \mathrm{~s}$, and $180 \mathrm{~s}$ after contrast media administration (iobitridol $350 \mathrm{mgI} / \mathrm{mL}$, Xenetix ${ }^{\circ}$, Guerbet, France) with weight-based dosing $(1.6 \mathrm{~mL} / \mathrm{kg})$.

The arterial phase was scanned using dual-energy $17 \mathrm{~s}$ after the attenuation of the abdominal aorta reached 80 Hounsfield unit (HU) at $100 \mathrm{kVp}$, using the care bolus technique of the vendor. For arterial phase dual-energy scanning, a tube potential pair of $80 / 150 \mathrm{kV}$ with a tin filter was used. The quality reference effective mAs was 
set to $250 \mathrm{mAs}$ for the $80-\mathrm{kV}$ tube and $125 \mathrm{mAs}$ for the $150-\mathrm{kV}$ tube. Detector configuration, gantry rotation time, and pitch were $192 \times 0.6 \mathrm{~mm}, 0.5 \mathrm{~s}$, and 0.6 , respectively. Images were reconstructed with semi-smooth quantitative body kernels in all phases.

\section{Quantitative imaging analysis}

The 80 - and $150-\mathrm{kV}$ images of the arterial phase were imported into the prototype software (eXamine: DE Tumor Segmentation, Siemens Healthineers, Forchheim, Germany). The software automatically creates three image sets: the arterial phase of the blended images with a mixed ratio of $0.6(60 \% 80 \mathrm{kV}$ and $40 \%$ tin-filtered $150 \mathrm{kV})$, virtual noncontrast, and material density iodine images. Image analysis was performed in mixed-volume images. Two fellowship-trained body radiologists (T.M.K. and S.J.P., 6 years of liver imaging experience) identified the HCC in the mixed-volume images and independently drew a region of interest (ROI) across the maximum dimension of the tumor in any plane (axial, coronal, or sagittal plane) (Fig. 1). Then the software automatically segmented the entire tumor volume and calculated the tumor volume and maximal diameter. In addition, the following were displayed: the mean $\mathrm{HU}$ of mixed-energy images, total iodine concentration, and vital iodine concentration. The total iodine concentration refers to the iodine per unit volume (in milligrams per milliliter) in the entire ROI, while vital iodine concentration refers to the iodine per unit volume in the only enhancing portion within the ROI. The tumor margin is manually edited if the tumor is not correctly segmented at once, by evaluating portal venous or equilibrium phase images. The sliceby-slice manual segmentation of the volumetric HCCs were also performed to compare with HCCs obtained by semiautomatic segmentation.

Subsequently, two intratumoral and two peritumoral layers from the tumor border were automatically generated using the peeling function with a layer thickness of $2 \mathrm{~mm}$ (outer layer 1, peritumoral region up to $2 \mathrm{~mm}$ from tumor margin; outer layer 2, peritumoral region from $2 \mathrm{~mm}$ to $4 \mathrm{~mm}$ from the tumor margin; inner layer 1, intratumoral region up to 2 $\mathrm{mm}$ from the tumor margin; inner layer 2 , intratumoral region from $2 \mathrm{~mm}$ to $4 \mathrm{~mm}$ from the tumor margin). In addition, five VOIs were generated with different volume coverage (tumor itself; $\mathrm{VOI}_{\mathrm{O} 1}$, tumor plus outer layer 1 ; $\mathrm{VOI}_{\mathrm{O} 2}$, tumor plus outer layers 1 and 2; $\mathrm{VOI}_{\mathrm{I} 1}$, tumor minus inner layer $1 ; \mathrm{VOI}_{\mathrm{I} 2}$, tumor minus inner layers 1 and 2) (Fig. 2). For the layers and VOIs outside the tumor, large vessels and areas outside the liver are manually removed.

An ROI was manually drawn in the aorta to calculate the normalized iodine concentration (NIC) to minimize intersubject variations. The software estimates the NIC by dividing vital iodine concentration of the ROI by the

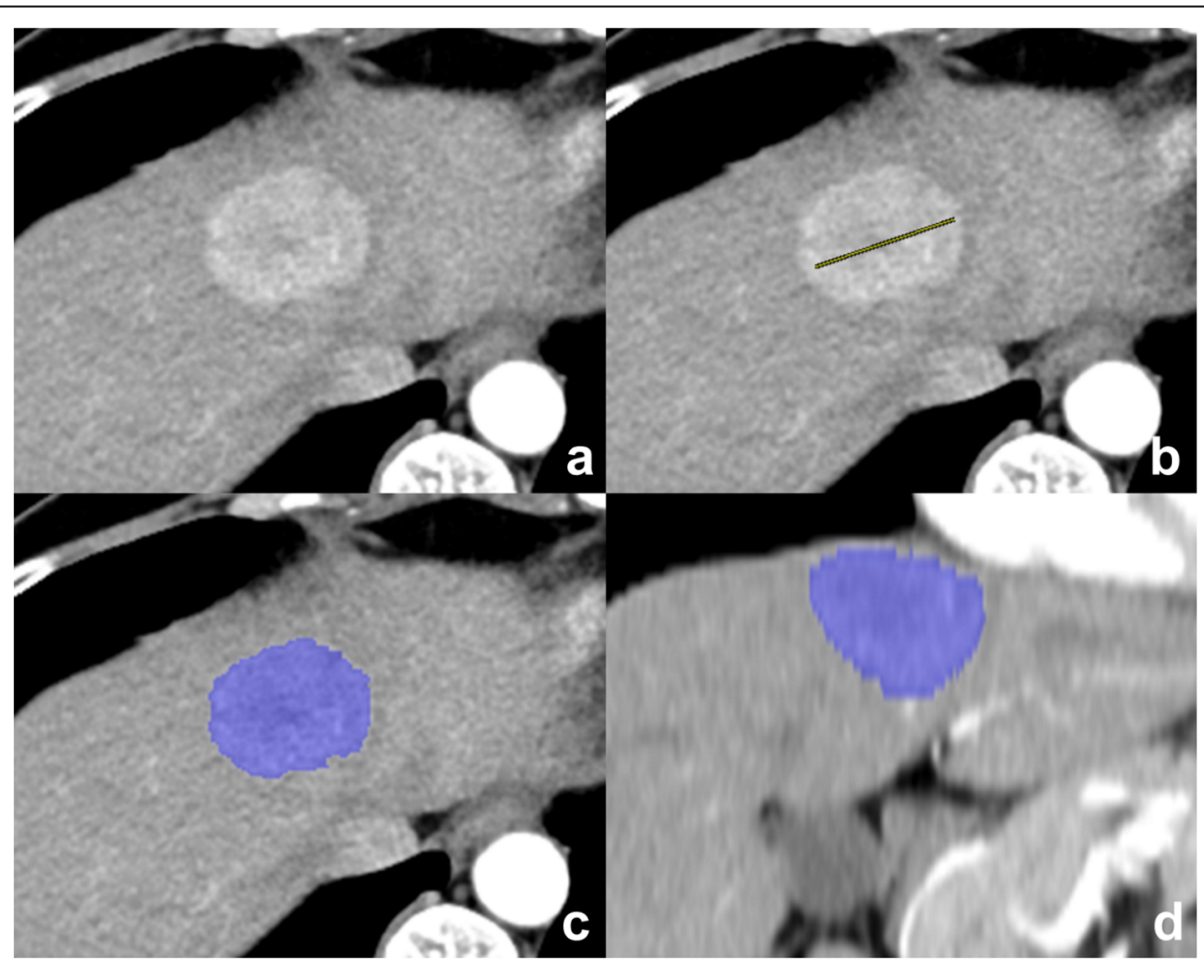

Fig. 1 Semiautomatic volumetric segmentation of the hepatocellular carcinoma (HCC). a HCC demonstrated in the mixed-energy images. b Readers manually draw a line across the maximum dimension of the tumor. $\mathbf{c}$ The software automatically segments the entire tumor volume. $\mathbf{d}$ Coronal reconstructed image shows the volumetric tumor segmented in three dimensions 


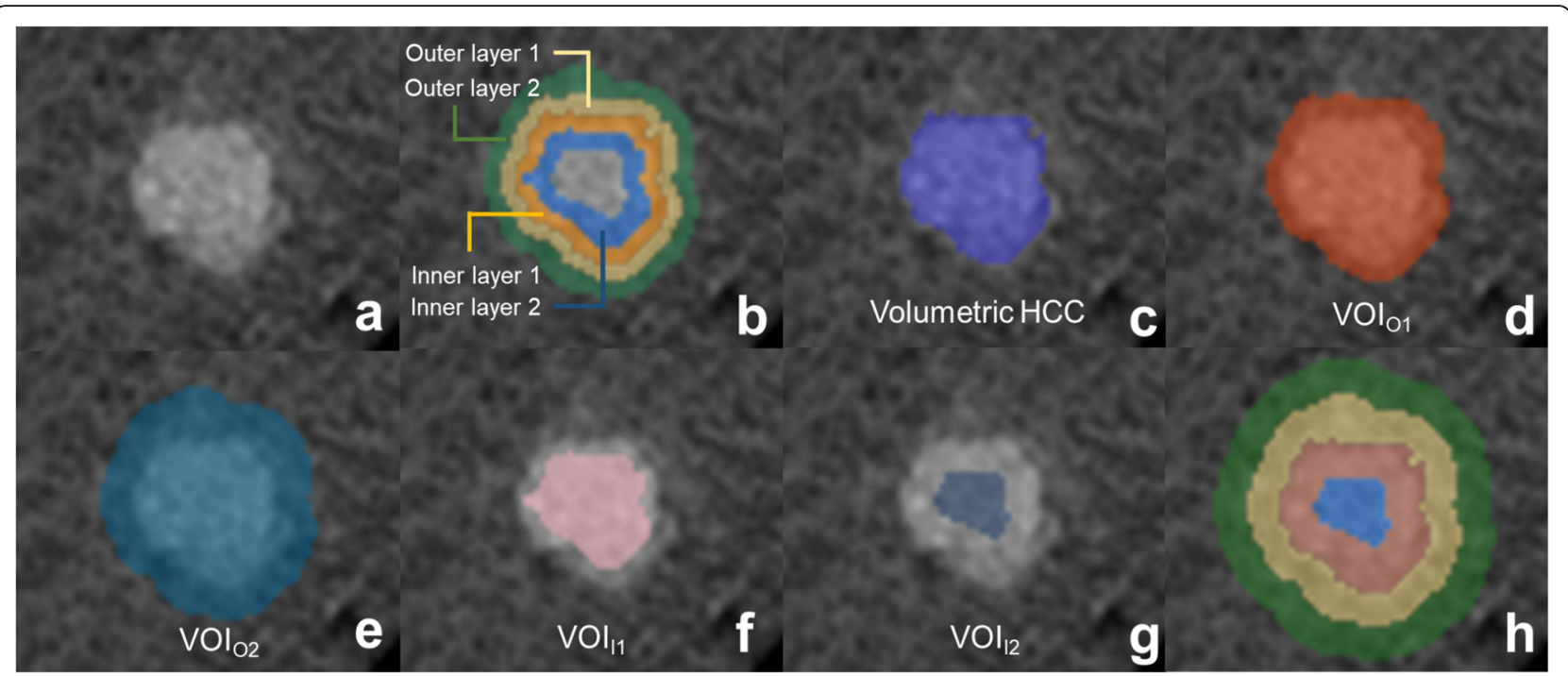

Fig. 2 Representative images of the layers and volume of interest (VOIs). a Original mixed-energy images. b Layer-by-layer analysis for two peritumoral layers (outer layer 1 [yellow], outer layer 2 [green]) and two intratumoral layers (inner layer 1 [orange], inner layer 2 [blue]) with a $2-$ $\mathrm{mm}$ layer thickness. $\mathbf{c} \mathrm{VOI}$ of the tumor. $\mathbf{d} \mathrm{VOl}_{\mathrm{O} 1}$, tumor plus outer layer 1. e $\mathrm{VOl}_{\mathrm{O}_{2}}$, tumor plus outer layers 1 and 2. $\mathbf{f} \mathrm{VOI}_{11}$, tumor minus inner layer 1. $\left.\mathbf{g ~ V O I}\right|_{2}$, tumor minus inner layers 1 and 2 . $\mathbf{h}$ The aforementioned analysis performed in the same manner with a 4-mm layer thickness

iodine concentration in the aorta. Additionally, the software automatically performs segmentation of the entire liver parenchyma to evaluate lesion-to-normal parenchyma ratios of mean attenuation by dividing the mean attenuation of the ROI into those of the normal liver parenchyma.

One radiologist (T.M.K., 6 years of liver imaging experience) blinded to the histologic findings performed the measurement. Additional measurements were performed in separate session by same radiologist (T.M.K.) at least 2 weeks apart and in one session by another radiologist (S.J.P., 6 years of liver imaging experience) to evaluate intraobserver and interobserver reliability, respectively. In addition, the aforementioned quantitative analysis was performed in the same manner but with different layer thicknesses $(4 \mathrm{~mm})$ to evaluate the effect of different peritumoral or intratumoral areas.

\section{Qualitative imaging analysis}

Two fellowship-trained body radiologists (T.M.K. and S.J.P., 6 years of liver imaging experience), who were blinded to the clinical and pathological data, independently assessed the images to evaluate the following qualitative features for MVI: (a) presence of peritumoral enhancement, which was defined as the existence of a detectable enhancing portion adjacent to the tumor border in the arterial phase, later becoming isoattenuation in the equilibrium phase [16, 17], and (b) tumor margins, whether they were smooth margins (defined as nodular tumors in all planes) or non-smooth margins (defined as nonnodular tumors in all planes) $[16,17]$. All HCCs were evaluated in each dynamic phase using different planes (axial, coronal, and sagittal planes). Disagreements were resolved by consensus. One reader (T.M.K.) repeated the abovementioned assessment in the same manner after 2 weeks to minimize the memory effect to evaluate intraobserver agreement.

\section{Outcomes}

Our primary end point was to evaluate the association between the NIC of peritumoral regions on DECT and MVI in HCC. Secondary end points were as follows: (a) to compare the diagnostic performance of peritumoral NIC with qualitative analysis by a human observer for identifying MVI, (b) to compare the diagnostic performance of peritumoral NIC with peritumoral mean attenuation for identifying MVI, and (c) to investigate the effect of different peritumoral areas $(2 \mathrm{~mm}$ vs. $4 \mathrm{~mm}$ ) on the diagnostic performance of MVI.

\section{Statistical analysis}

To compare parameters between the HCCs with and without MVI, continuous data were assessed using the Mann-Whitney test, while categorical variables were analyzed using the chi-square test. Additionally, to investigate the association between subjective peritumoral enhancement and DECT parameters of peritumoral layers, the mean attenuation and layer-to-normal parenchyma ratio of mean attenuation of peritumoral layers were compared between the groups with absent and present peritumoral enhancement. A receiver operating characteristic (ROC) curve analysis was performed using quantitative parameters of layers and VOIs and qualitative parameters to determine the diagnostic performance 
for the prediction of MVI. Areas under the ROC curves (AUCs) with 95\% confidence intervals (CIs) were calculated for the significant CT parameters, and a comparison analysis of AUCs was performed. Variables with $P<0.10$ in univariate analysis were applied to binary logistic regression analysis. Continuous variables were converted to categorical variables with optimal cutoff values to perform multivariate analysis. Among the NIC of multiple layers and VOIs, only one layer or VOI that showed the highest AUC was included in the multivariate analysis to avoid multicollinearity of parameters.

Intraobserver and interobserver agreements were evaluated using intraclass coefficient class (ICC) and Cohen $\mathrm{k}$-statistics. The agreements of DECT parameters in volumetric HCCs between manual and semiautomatic segmentation, and agreements of maximal diameter of HCCs obtained in preoperative CT by both segmentation techniques and histopathology were also evaluated using ICC. The strength of agreement via ICC and $\mathrm{k}$ values $<0.4,0.4-0.6,0.6-0.8$, and $>0.8$ were categorized poor, moderate, good, and excellent agreement, respectively. Statistical analyses were performed using a statistical software package (SPSS version 23, SPSS, Inc., Chicago, IL). Differences of $P<0.05$ were considered statistically significant.

\section{Results}

\section{Patient characteristics}

A total of 47 patients with $\mathrm{HCC}$ underwent preoperative liver CT within 4 weeks of hepatic resection. Among them, 11 patients were excluded because of preoperative transarterial chemoembolization $(n=7)$, small size $(<1$ $\mathrm{cm})$ of the HCC $(n=2)$, and macrovascular invasion $(\mathrm{n}=2)$. Finally, 36 patients $(\mathrm{men} /$ women $=24: 12$, mean age $59.9 \pm 8.5$ years) with MVI $(n=14)$ and without MVI $(n=22)$ were included. The median tumor size was 2.7 $\mathrm{cm}$ (range, $1.2-4.5 \mathrm{~cm}$ ). The surgical procedures included segmentectomy $(n=23)$, right anterior sectionectomy $(n=1)$, left lateral sectionectomy $(n=3)$, right hemihepatectomy $(n=2)$, left hemihepatectomy $(n=3)$, and total hepatectomy for transplantation $(n=4)$. The mean time between dual-energy CT and surgery was 16 days (range, 1-30 days). The detailed information of the study group is summarized in Table 1.

Between the two groups with and without MVI, no significant difference was observed in age, sex, histologic grade of hepatic fibrosis, underlying liver disease, and Edmondson-Steiner grade of HCCs $(P>0.05$, Table 1$)$. Tumor size did not differ between the two groups with and without MVI $(3 \pm 1.3 \mathrm{~cm}$ vs. $2.5 \pm 0.9 \mathrm{~cm}, P=0.17)$. The group with MVI showed significantly higher serum alpha-fetoprotein (AFP) levels (median $8.9 \mathrm{ng} / \mathrm{mL}$, range 2.1-2022.4 $\mathrm{ng} / \mathrm{mL}$ ) compared with those without MVI (median $3.0 \mathrm{ng} / \mathrm{mL}$, range $0.9-1387.3 \mathrm{ng} / \mathrm{mL}, P=0.03$ ).
Table 1 Clinicopathological characteristics of the patients

\begin{tabular}{|c|c|c|c|}
\hline Characteristics & $\begin{array}{l}\text { MVI }(-) \\
(n=22, \%)\end{array}$ & $\begin{array}{l}\text { MVI (+) } \\
(n=14, \%)\end{array}$ & $P$-value** \\
\hline \multicolumn{4}{|l|}{ Age (years) } \\
\hline Median (range) & $61.5(35-74)$ & $56.5(46-73)$ & 0.4 \\
\hline \multicolumn{4}{|l|}{ Sex } \\
\hline Men & $14(63.6)$ & $10(71.4)$ & 0.6 \\
\hline Women & $8(36.4)$ & $4(28.6)$ & \\
\hline \multicolumn{4}{|l|}{$\begin{array}{l}\text { Staging of hepatic } \\
\text { fibrosis }^{a}\end{array}$} \\
\hline 0 & 1 & 0 & 0.6 \\
\hline 1 & 1 & 1 & \\
\hline 2 & 5 & 3 & \\
\hline 3 & 6 & 3 & \\
\hline 4 & 9 & 7 & \\
\hline
\end{tabular}

Underlying liver disease

$\begin{array}{lll}\text { Hepatitis B } & 16(72.7) & 12(85.7) \\ \text { Hepatitis C } & 2(9.1) & 2(14.3) \\ \text { Alcoholism } & 2(9.1) & 0(0) \\ \text { Non B Non C } & 2(9.1) & 0(0)\end{array}$

Edmondson-Steiner

grade of HCC

$\begin{array}{cccc}\text { I } & 7 & 1 & 0.06 \\ \text { II } & 12 & 7 & \\ \text { III } & 3 & 6 & \\ \text { AFP level }(\mathrm{ng} / \mathrm{ml}) & & \end{array}$

Median (range) $\quad 3.02(0.9-1387.3) \quad 8.89(2.11-2022.37) \quad 0.03$ PIVKA-Il level (mAU/ml)

Median (range) 32 (16-827) $66(0.74-6168) \quad 0.09$

Abbreviations: MVI microvascular invasion, AFP Alpha-fetoprotein, PIVKA-II Protein induced by vitamin $\mathrm{K}$ absence or antagonist-II, $S D$ standard deviation ${ }^{\mathrm{a} A}$ staging system for chronic hepatitis by the Korean Study Group for the Pathology of Digestive Diseases was used (0, no fibrosis; 1 , portal fibrosis; 2 , periportal fibrosis, 3, septal fibrosis; 4, cirrhosis)

** Continuous data were assessed using Mann-Whitney test while categorical variables were analyzed using chi square test. Staging of hepatic fibrosis, underlying liver disease, Edmondson-Steiner grade of HCC were compared as follows; stage 4 vs. stage $0-3$, hepatitis B vs. others, grade III vs. grade I-II

\section{Comparisons of volumetric DECT parameters between groups with and without $\mathrm{MVI}$}

The NICs of outer layer 1, outer layer 2, $\mathrm{VOI}_{\mathrm{O} 1}$, and $\mathrm{VOI}_{\mathrm{O} 2}$ were significantly higher in the MVI group than in the MVI absent group $(P<0.05$ for all, Table 2$)$. Other layers and VOIs showed no significant differences in NIC values between the two groups $(P>0.05)$.

For entire volumetric $\mathrm{HCCs}$, quantitative parameters including total volume, maximal diameter, mean $\mathrm{HU}$ in mixed-energy images, lesion-to-normal parenchyma ratio of mean attenuation value, total iodine concentration, and NIC showed no difference between HCCs with and without MVI $(P>0.05)$. 
Table 2 The normalized iodine concentrations of peritumoral and intratumoral regions between MVI absent and MVI present groups

\begin{tabular}{lllll}
\hline $\begin{array}{llll}\text { Layer } \\
\text { thickness }\end{array}$ & Region* & $\mathrm{NIC}(\mathrm{mg} / \mathrm{ml})$ & & \\
\cline { 3 - 5 } & & $\mathrm{MVI}(-)(n=22)$ & $\mathrm{MVI}(+)(n=14)$ & $P$-value \\
\hline $2 \mathrm{~mm}$ & Outer layer 1 & $0.07 \pm 0.03$ & $0.10 \pm 0.03$ & 0.01 \\
& Outer layer 2 & $0.05 \pm 0.03$ & $0.07 \pm 0.03$ & 0.04 \\
& Inner layer 1 & $0.12 \pm 0.07$ & $0.16 \pm 0.04$ & 0.10 \\
& Inner layer 2 & $0.15 \pm 0.08$ & $0.19 \pm 0.07$ & 0.17 \\
& VOI O1 & $0.11 \pm 0.05$ & $0.14 \pm 0.03$ & 0.02 \\
& VOI $_{\text {O2 }}$ & $0.09 \pm 0.04$ & $0.12 \pm 0.03$ & 0.02 \\
& VOI $_{11}$ & $0.15 \pm 0.08$ & $0.19 \pm 0.07$ & 0.19 \\
\hline
\end{tabular}

Abbreviations: MVI microvascular invasion, NIC normalized iodine concentration, $\mathrm{VOI}$ volume of interest

Values are presented in means \pm standard deviations

*Data which was obtained in part of the patient group was excluded $\left(\mathrm{VOI}_{12}, n=33\right)$

\section{Diagnostic performance of volumetric DECT parameters versus qualitative features for identifying MVI}

The AUCs of NICs of layers and VOIs ranged from 0.67 to 0.75 (Table 3, Fig. 3). The largest AUC was obtained in the NIC of outer layer 1 (0.75). A sensitivity of $78.6 \%$ and specificity of $68.2 \%$ were obtained at cutoff values of 0.082 . There were no significant differences between areas in a pairwise comparison of ROC curves among layers and VOIs (all $P>0.05$ ).

Peritumoral enhancement of HCCs was present in $36.4 \%(8 / 22)$ of HCCs without MVI and in $64.3 \%$ (9/14) of HCCs with MVI. The MVI absent group showed nonsmooth tumor margins in $45.5 \%(10 / 22)$, while the MVI present group showed $71.4 \%$ (10/14). There were no significant differences between the groups with and without MVI in peritumoral enhancement and non-smooth tumor margins $(P=0.10,0.13)$. The AUCs of peritumoral enhancement and non-smooth tumor margin were 0.64 (95\% CI 0.45-0.83) and 0.63 (95\% CI 0.44-0.82), respectively. The AUCs of the NICs and qualitative image features showed no significant differences $(P>0.05)$.

\section{Multivariate analysis of clinicopathological findings and peritumoral iodine concentration for identifying MVI} In univariate analysis, the MVI group showed more frequent abnormal protein induced by vitamin $\mathrm{K}$ absence or antagonist-II (PIVKA-II) level $(P=0.05)$, larger maximal diameter $(P=0.04)$, and higher NIC of outer layer $1 \quad(P=0.02)$ than the MVI absent group. In multivariate analysis, NIC of outer layer 1 was the only independent factor for predicting MVI, with an odds ratio of $7.14(P=0.04$, Figs. 4 and 5$)$. The serum PIVKA-II level, Edmondson-Steiner grade, and maximal diameter were not significant predictive factors in both sets of multivariate regression analyses $(P>0.05$, Table 4$)$.

\section{Relationship between peritumoral enhancement and volumetric DECT parameters of the peritumoral layers} Layer-to-normal parenchyma ratio of mean HU values was significantly higher in outer layer 2 , in both $2-\mathrm{mm}$ and 4-mm layer thickness, in HCCs with peritumoral enhancement than in those in HCCs without peritumoral enhancement $(P=0.03, P=0.04$, respectively). However, the mean $\mathrm{HU}$ and NIC showed no significant difference between the two groups $(P>0.05$, Supplementary Table 1, Additional file 1).

\section{Diagnostic performance of peritumoral NICs versus peritumoral CT attenuations for identifying $\mathrm{MVI}$}

The mean attenuations of all layers and VOIs showed no significant difference between the two groups $(P>$ 0.05, Supplementary Table 2, Additional file 2). The AUCs of the mean attenuations of layers and VOIs ranged from 0.56 to 0.66 . They were not significantly different from the AUCs of NICs in pairwise comparisons (all $P>0.05$ ).

\section{Effect of layer thickness}

In the 4-mm layer thickness, the NICs of outer layer 1, $\mathrm{VOI}_{\mathrm{O} 1}$, and $\mathrm{VOI}_{\mathrm{O} 2}$ were also significantly higher in the MVI group than in the MVI absent group (all $P<0.05$, Supplementary Table 3, Additional file 3). The AUCs of the NICs ranged from 0.69 to 0.75 . The largest AUC was obtained for $\mathrm{VOI}_{\mathrm{O} 2}$ (AUC 0.75). There were no significant differences between areas in a pairwise comparison of ROC curves, including the values obtained in 2-mm layer thickness (all $P>0.05$ ).

Table 3 The diagnostic performance of normalized iodine concentrations (NICs) of peritumoral and intratumoral regions and qualitative features for predicting MVI

\begin{tabular}{|c|c|c|c|c|c|c|c|c|c|}
\hline & \multicolumn{7}{|l|}{$\mathrm{NIC}^{*}$} & \multicolumn{2}{|c|}{ Qualitative features } \\
\hline & Outer layer 1 & Outer layer 2 & Inner layer 1 & Inner layer 2 & $\mathrm{VOI}_{\mathrm{O} 1}$ & $\mathrm{VOl}_{\mathrm{O} 2}$ & $\mathrm{VOI}_{11}$ & $\begin{array}{l}\text { Peritumoral } \\
\text { enhancement }\end{array}$ & $\begin{array}{l}\text { Non-smooth } \\
\text { tumor margins }\end{array}$ \\
\hline$\overline{A \cup C}$ & 0.75 & 0.71 & 0.70 & 0.68 & 0.73 & 0.74 & 0.67 & 0.64 & 0.63 \\
\hline $95 \% \mathrm{Cl}$ & $0.58-0.91$ & $0.53-0.89$ & $0.52-0.87$ & $0.50-0.85$ & $0.57-0.90$ & $0.58-0.91$ & $0.49-0.85$ & $0.45-0.83$ & $0.44-0.82$ \\
\hline
\end{tabular}

Abbreviations: AUC area under the ROC curve, $\mathrm{Cl}$ confidence interval, $\mathrm{VOI}$ volume of interest

* Data which was obtained in part of the patient group was excluded $\left(\mathrm{VOI}_{12}, n=33\right)$ 

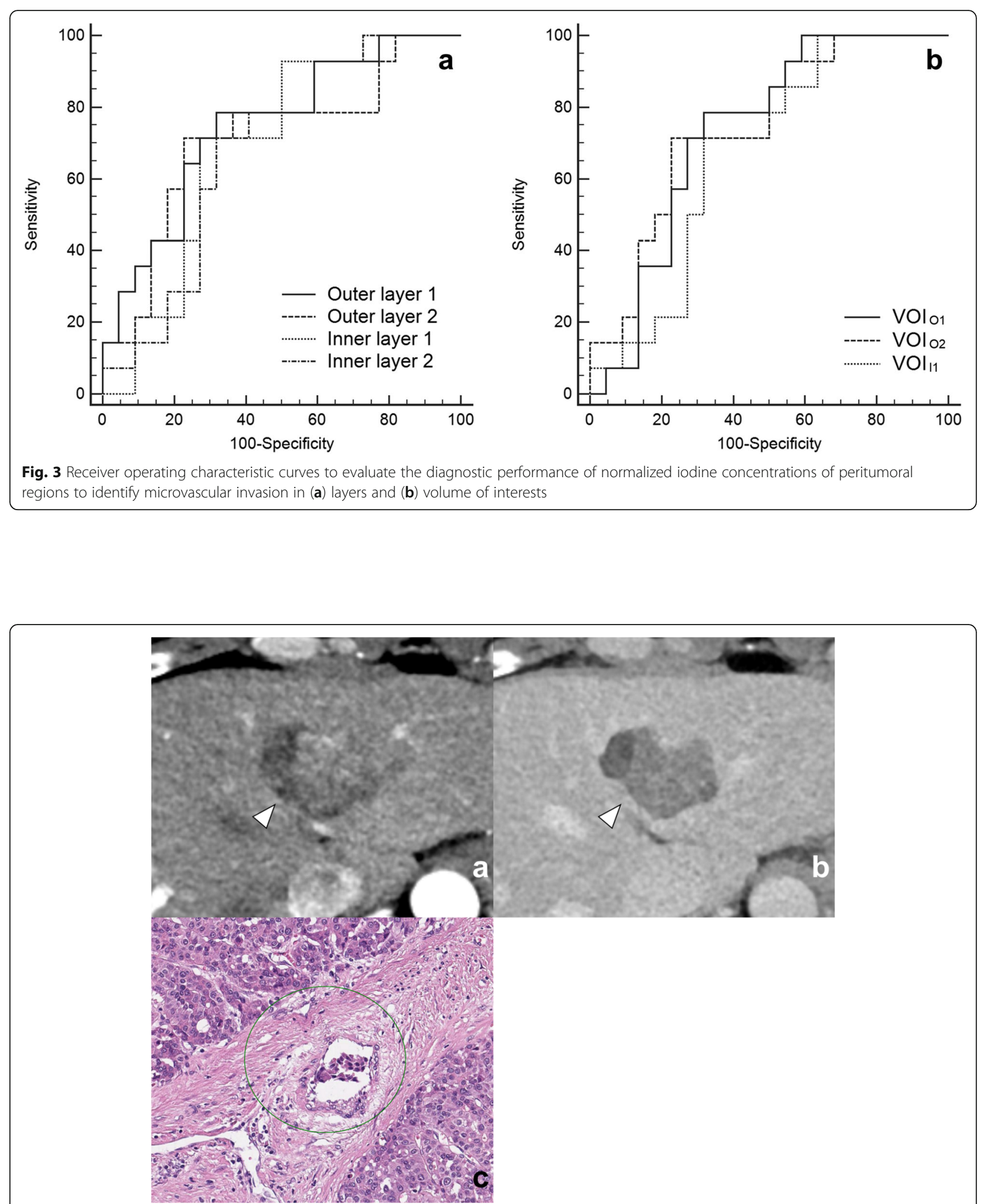

Fig. 4 A 54-year-old man with hepatocellular carcinoma with microvascular invasion (MVI). a No definite peritumoral enhancement found in the arterial phase compared with $\mathbf{b}$ equilibrium phase images. However, the NIC of the peritumoral layer with a 2-mm distance from the tumor margin is $0.096 \mathrm{mg} / \mathrm{mL}$, which is higher than the cutoff value for predicting MVI of $0.082 \mathrm{mg} / \mathrm{mL}$. c Microscopic evaluation reveals a tumor cells (circle) within the small vessel (hematoxylin-eosin stain, original magnification: $\times 20$ and $\times 20$ ) 


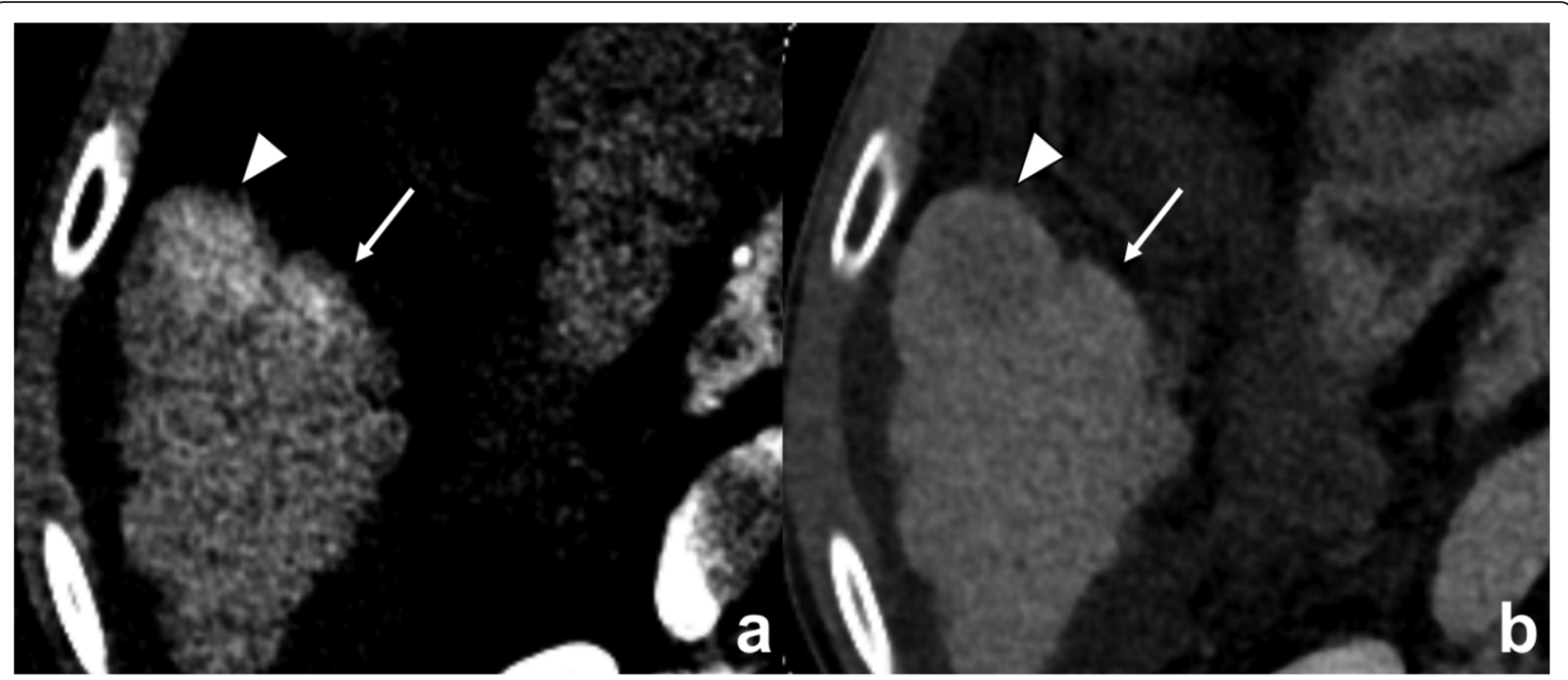

Fig. 5 A 64-year-old man with hepatocellular carcinoma (HCC) without microvascular invasion (MVI). a Enhancing area found in the peritumoral area (arrows) adjacent to the HCC (arrowheads) in the arterial phase, which became $\mathbf{b}$ isoattenuation in the equilibrium phase. However, the NIC of the peritumoral layer with a $2-\mathrm{mm}$ distance from the tumor margin is $0.03 \mathrm{mg} / \mathrm{mL}$, lower than $0.082 \mathrm{mg} / \mathrm{mL}$, which is the cutoff value for identifying $\mathrm{MVI}$

\section{Comparison of volumetric HCC between manual} segmentation and semiautomatic segmentation

The agreements of DECT parameters of volumetric HCCs between two segmentation techniques were excellent $(I C C=$ 0.97-1.0, Supplementary Table 4, Additional file 4). Maximal diameter of HCCs in histopathology $(26.7 \pm 10.8 \mathrm{~mm})$ showed excellent correlation with those of HCCs in preoperative CT by both manual $(27.2 \pm 9.8 \mathrm{~mm}, I C C=0.94)$ and semiautomatic $(28.3 \pm 10.7 \mathrm{~mm}, I C C=0.91)$ segmentation methods.

\section{Intra- and interobserver agreements}

Interobserver and intraobserver agreements were excellent in all measured quantitative parameters $(I C C=0.81-1.0,0.85-$
1.0). In qualitative parameters, interobserver and intraobserver agreements were good to excellent and excellent, respectively $(\kappa=0.67-0.71,0.78-0.89$, Supplementary Table 5, Additional file 5).

\section{Discussion}

Our study demonstrated that the NIC of the peritumoral zones and intratumoral zones of HCCs measured by DECT was useful for the prediction of MVI. In more detail, outer layer 1 and outer layer 2, which corresponded to the peritumoral layers, showed significantly higher NIC in the MVI present group than in the MVI absent group. Interestingly, the combination of HCCs and peritumoral

Table 4 Univariate and multivariate analyses of clinical and CT parameters in predicting MVI

\begin{tabular}{|c|c|c|c|c|}
\hline \multirow[t]{2}{*}{ Parameters } & \multicolumn{2}{|l|}{ Univariate analysis } & \multicolumn{2}{|l|}{ Multivariate analysis } \\
\hline & Odds ratio $(95 \% \mathrm{Cl})$ & $P$-value & Odds ratio $(95 \% \mathrm{Cl})$ & $P$-value \\
\hline Age (> 60 years) & $0.63(0.16-2.41)$ & 0.50 & & \\
\hline Sex (Female) & $0.70(0.16-2.98)$ & 0.63 & & \\
\hline Staging of hepatic fibrosis (Stage 4) & $1.44(0.38-5.57)$ & 0.59 & & \\
\hline $\operatorname{AFP}(>8.7 \mathrm{ng} / \mathrm{ml})$ & $2.67(0.65-10.88)$ & 0.17 & & \\
\hline PIVKA-II (> 40 mAU/mL) & $4.38(1.03-18.63)$ & 0.05 & $2.71(0.47-15.58)$ & 0.26 \\
\hline Edmondson-Steiner grade of HCC (III) & $4.75(0.95-23.85)$ & 0.06 & $5.64(0.67-47.25)$ & 0.11 \\
\hline Total volume of HCC (> $4.5 \mathrm{ml}$ ) & $2.16(0.54-8.57)$ & 0.27 & & \\
\hline Maximal diameter of HCC (> $32 \mathrm{~mm}$ ) & $4.53(1.06-19.41)$ & 0.04 & $4.04(0.67-24.37)$ & 0.13 \\
\hline Peritumoral enhancement & $3.15(0.78-12.73)$ & 0.11 & & \\
\hline Nonsmooth tumor margin & $3.00(0.72-12.55)$ & 0.13 & & \\
\hline NIC of outer layer 1 (>0.082 mg/ml) & $6.42(1.37-30.05)$ & 0.02 & $7.14(1.12-45.40)$ & 0.04 \\
\hline
\end{tabular}

Abbreviations: MVI microvascular invasion, AFP Alpha-fetoprotein, PIVKA-II Protein induced by vitamin $\mathrm{K}$ absence or antagonist-II, NIC normalized iodine concentration, $\mathrm{VOI}$ volume of interest, $\mathrm{Cl}$ confidence interval 
regions $\left(\mathrm{VOI}_{\mathrm{O} 1}, \mathrm{VOI}_{\mathrm{O} 2}\right)$ also showed different $\mathrm{NIC}$ values between the MVI present and absent groups with comparable AUCs to those of peritumoral regions only. This might imply that MVI status could be predicted by analyzing NICs of both intratumoral and peritumoral regions as well as peritumoral regions only. We also found that the $\mathrm{NIC}$ of the peritumoral region up to $2 \mathrm{~mm}$ from the tumor margin was an independent predictive factor for MVI in multivariate analysis. Yang et al. indicated that the NICs of HCCs with MVI were significantly higher than those of HCCs without MVI [18]. However, our results showed no significant difference in the NICs of HCCs between the two groups. This discrepancy may have contributed to the difference in the way ROI was placed. A previous study drew the ROI with a diameter of half of the tumor size in one axial image, whereas we segmented the whole volume of the HCCs in three dimensions, which better reflects the nature of the tumor.

Peritumoral enhancement is usually defined as grossly contrast material enhancement outside of the tumor border at the arterial phase that becomes isointense with background liver parenchyma in the later dynamic phase images [19]. This imaging finding probably relates to the known hypothesis of hemodynamic perfusion changes existing in compensatory arterial hyperperfusion, which can occur in the region of decreased portal flow caused by minute portal branch occlusion because of tumor thrombi, assuming that the draining veins of HCCs are usually portal venules [20]. Until now, there has been controversy as to whether there is a correlation between peritumoral enhancement in CT and MVI of HCCs. Previous studies have demonstrated that peritumoral enhancement significantly increases the risk of MVI, and the area of peritumoral hemodynamic change in HCC patients with microscopic portal invasion was significantly larger than those without it in combined CT hepatic arteriography and CT arterioportography [21, 22]. However, another study by Chou et al. described peritumoral enhancement in CT was not a significant risk factor for MVI [16]. One of the potential reasons for these discrepancies among the previous studies could be the qualitative assessment of peritumoral enhancement, ROI measurement of attenuation coefficient, and heterogeneous scanning parameters. Although qualitative image findings such as peritumoral enhancement and nonsmooth tumor margins showed similar AUCs compared with those of NICs in our study, there were no significant factors in univariate analysis for predicting MVI, in contrast to previous studies $[7,9,16,17,22]$. The number of patients might be insufficient to evaluate these multiple qualitative imaging features. However, the NIC of the peritumoral layer was an independent predictive factor for MVI in this setting, which suggests an additional value for MVI prediction in preoperative imaging.
The diagnostic accuracy of our study (AUC 0.67-0.75, sensitivity $78.6 \%$, specificity $68.2 \%$ ) was comparable to that of a recent study that evaluated peritumoral enhancement qualitatively in CT (accuracy 74.3\%) [22].

In our study, despite the attenuation coefficients of peritumoral areas showed lower AUCs (0.56-0.66) than peritumoral NICs $(0.67-0.75)$, there was no statistical difference. It might be contributed to the small study population. However, in contrast to peritumoral NICs, there were no significant factors for the prediction of MVI in peritumoral attenuation coefficients. A previous study revealed that dual-energy CT demonstrated a linear relationship with low relative error (less than 10\%) between measured and actual iodine concentrations in an in vitro experiment [23]. Based on our study results, we suggest that the iodine concentration calculated using dual-energy CT may reflect peritumoral perfusional changes caused by microcirculation of the blood flow more accurately than attenuation measurements.

A recent study demonstrated radiomic analysis of contrast-enhanced CT for predicting MVI [22]. They integrated the radiomic features of peritumoral and intratumoral region with clinical factors and radiographic features, and this predictive model achieved high accuracy in estimating MVI with an AUC of 0.89. In this study, they found the radiomic features of peritumoral zone at $5 \mathrm{~mm}$ distance from the tumor surface differ according to the presence of MVI. Our results are in concordance with this study, in that the quantitative characteristics of the peritumoral area were different depending on the presence or absence of MVI. One thing we should point out is, the patients in our study have variable degree of liver fibrosis. According to previous literatures, with hepatic fibrogenesis, microcirculatory changes results in increased total hepatic resistance with altered portal venous blood flow, compensated by increased hepatic arterial flow [24, 25]. Liver perfusion decreases while arterial perfusion increases in patients with cirrhosis, and correlates with severity [26]. The presence of liver cirrhosis (stage 4 of hepatic fibrosis) were not significantly different between MVI present and absent group, but heterogeneity of fibrosis degree might change the enhancement characteristics of the peritumoral parenchyma and subsequently affect both quantitative and qualitative analysis of peritumoral region.

The main issue of our study was how to correctly draw the tumor boundary. The initial process for tumor segmentation is the most important step because it affects all of the peritumoral layers and intratumoral layers in the following process. We used a dedicated software prototype (eXamine: "DE Tumor Segmentation") that allows for a refined volumetric semiautomatic segmentation of the entire tumor and a respective evaluation of 
spectral data. This application is designed to evaluate different parameters of a tumor scanned with Siemens DECT technology using an iodine contrast agent. It also provides semiautomatic segmentation of the tumor peel datasets. Once the user selects the number of peels in the prototype, the user obtains the respective number of peels to the outside and inside of the original tumor automatically and a respective analysis of the different layers from a spectral perspective. We additionally performed manual segmentation of HCCs to evaluate the performance of semiautomatic segmentation, and found high agreement between the DECT parameters of HCCs in both segmentation techniques (ICC 0.97-1.0). One remarkable observation in our study is that we found strong correlations between the macroscopic diameter of the surgical tumors and the maximal diameter of HCCs obtained by semiautomatic segmentation (ICC 0.91), as well as manual segmentation (ICC 0.94). These results suggest, although we had to edit the tumor margins manually in a few HCCs with ill-defined margins, semiautomatic segmentation technique used in the current study can perform tumor segmentation accurately and efficiently. It minimized the hand-related artifacts and showed excellent intra- and interobserver agreements. The manual drawing in each slice was also accurate and simple but takes a lot of time and effort. Our study is in concordance with previous studies that demonstrated good correlation between manual and semiautomatic segmentation, while latter technique can reduce the time for measurement and show excellent accuracy and repeatability [27-30]. This technique can also be applied to the segmentation of tumors other than HCCs. The layer thickness $(2 \mathrm{~mm}$ or $4 \mathrm{~mm})$ did not affect the diagnostic performance in our study. This result suggests that perfusional change occurs in the peritumoral region within a distance of at least $8 \mathrm{~mm}$ from the tumor margin.

This study has some limitations. First, this retrospective study was performed in a single center with a small number of patients. Additionally, only surgically confirmed HCCs were included in this study. A potential selection bias might have occurred. However, because MVI can only be confirmed by pathology, strict inclusion criteria are inevitable. Second, tumors less than 1 $\mathrm{cm}$ were excluded from our study because of the potential measurement error. Although it was not intended, all HCCs of the patients were less than $5 \mathrm{~cm}$ in maximal diameter. Therefore, our results cannot be generalized to small $(<1 \mathrm{~cm})$ or large $(>5 \mathrm{~cm})$ HCCs. Third, various degree of liver fibrosis in our study population may affect the enhancement characteristics of peritumoral region and results of our study. A larger study with subgroup analysis according to the fibrosis degree would be helpful to decrease this bias. Fourth, the software used in our study was only available in dual-source dual-energy CT. Other dual-energy techniques, such as rapid $\mathrm{kVp}$ switching or dual-layer spectral CT, were not used in our study. Further studies using other dual-energy CT vendors are warranted.

\section{Conclusion}

In conclusion, the volumetric iodine quantification of peritumoral regions in arterial phase may be useful for predicting MVI in preoperative dual-energy CT.

\section{Supplementary information}

Supplementary information accompanies this paper at https://doi.org/10. 1186/s40644-020-00338-7.

Additional file 1: Table S1. Relationship between peritumoral enhancement and quantitative parameters of peritumoral layers.

Additional file 2: Table S2. The Hounsfield units of peritumoral and intratumoral regions between MVI absent and MVI present groups.

Additional file 3: Table S3. The normalized iodine concentration (NICs) of peritumoral and intratumoral regions in $4 \mathrm{~mm}$ layer thickness between MVI absent and MVI present groups.

Additional file 4: Table S4. Comparison of semiautomatic segmentation and manual segmentation.

Additional file 5: Table S5. Intraobserver and interobserver agreements of quantitative and qualitative parameters.

\section{Abbreviations}

CT: Computed tomography; DECT: Dual-energy computed tomography; HCC: Hepatocellular carcinoma; MVI: Microvascular invasion; NIC: Normalized iodine concentration; VOI: Volume of interest

\section{Acknowledgements}

We would like to thank Bernhard Schmidt, Sedlmair Martin, and Seong Yong Pak for developing and updating the software used in the current study.

\section{Authors' contributions}

JML, IJ, SKJ contributed the study concept and design. TMK and SJP analyzed and interpreted the patient data. BS and SM made the software program for the image analysis. TMK, JHY, IJ, JML were major contributor in writing and revising the manuscript. All authors read and approved the final manuscript.

Funding

We have no funding information to disclose.

\section{Availability of data and materials}

All data generated or analysed during this study are included in this published article [and its supplementary information files].

Ethics approval and consent to participate

The institutional review board of Seoul National University Hospital approved this retrospective study and waived the requirement for informed consent.

\section{Consent for publication}

Not applicable.

\section{Competing interests}

Author Bernhard Schmidt and Sedlmair Martin are employees of Siemens Healthineers Ltd. However, control of all data and information submitted for publication was given to the authors who were not affiliated with Siemens Healthineers Ltd. For the remaining authors, they have nothing to disclose.

\section{Author details}

${ }^{1}$ Department of Radiology, Seoul National University Hospital, Seoul, South Korea. ${ }^{2}$ Department of Radiology, Seoul National University College of 
Medicine, Seoul, South Korea. ${ }^{3}$ Institute of Radiation Medicine, Seoul National University Medical Research Center, Seoul, South Korea. ${ }^{4}$ Research and development department, Siemens Healthineers, Forchheim, Germany.

Received: 22 May 2020 Accepted: 7 August 2020

Published online: 18 August 2020

\section{References}

1. Omata M, Cheng AL, Kokudo N, Kudo M, Lee JM, Jia J, et al. Asia-Pacific clinical practice guidelines on the management of hepatocellular carcinoma: a 2017 update. Hepatol Int. 2017;11(4):317-70.

2. Bruix J, Gores GJ, Mazzaferro V. Hepatocellular carcinoma: clinical frontiers and perspectives. Gut. 2014;63(5):844-55.

3. Llovet JM, Schwartz M, Mazzaferro V. Resection and liver transplantation for hepatocellular carcinoma. Semin Liver Dis. 2005;25(2):181-200.

4. Zimmerman MA, Ghobrial RM, Tong MJ, Hiatt JR, Cameron AM, Hong J, et al. Recurrence of hepatocellular carcinoma following liver transplantation: a review of preoperative and postoperative prognostic indicators. Arch Surg. 2008;143(2):182-8.

5. Imamura H, Matsuyama Y, Tanaka E, Ohkubo T, Hasegawa K, Miyagawa S, et al. Risk factors contributing to early and late phase intrahepatic recurrence of hepatocellular carcinoma after hepatectomy. J Hepatol. 2003; 38(2):200-7.

6. Ahn SY, Lee JM, Joo I, Lee ES, Lee SJ, Cheon GJ, et al. Prediction of microvascular invasion of hepatocellular carcinoma using gadoxetic acidenhanced MR and (18)F-FDG PET/CT. Abdom Imaging. 2015:40(4):843-51.

7. Renzulli M, Brocchi S, Cucchetti A, Mazzotti F, Mosconi C, Sportoletti C, et al. Can current preoperative imaging be used to detect microvascular invasion of hepatocellular carcinoma? Radiology. 2016;279(2):432-42.

8. Ariizumi S-i, Kitagawa K, Kotera Y, Takahashi Y, Katagiri S, Kuwatsuru R, et al. A non-smooth tumor margin in the hepatobiliary phase of gadoxetic acid disodium (Gd-EOB-DTPA)-enhanced magnetic resonance imaging predicts microscopic portal vein invasion, intrahepatic metastasis, and early recurrence after hepatectomy in patients with hepatocellular carcinoma. J Hepatobiliary-Pancreat Sci. 2011;18(4):575-85.

9. Segal E, Sirlin CB, Ooi C, Adler AS, Gollub J, Chen X, et al. Decoding global gene expression programs in liver cancer by noninvasive imaging. Nat Biotechnol. 2007;25(6):675-80.

10. Kim JH, Joo I, Lee JM. Atypical Appearance of Hepatocellular Carcinoma and Its Mimickers: How to Solve Challenging Cases Using Gadoxetic AcidEnhanced Liver Magnetic Resonance Imaging. Korean J Radiol. 2019;20(7): 1019-41.

11. Goo HW, Goo JM. Dual-Energy CT: New horizon in medical imaging. Korean J Radiol. 2017;18(4):555-69.

12. Agrawal MD, Pinho DF, Kulkarni NM, Hahn PF, Guimaraes AR, Sahani DV. Oncologic applications of dual-energy $C T$ in the abdomen. RadioGraphics. 2014;34(3):589-612.

13. De Cecco CN, Darnell A, Rengo M, Muscogiuri G, Bellini D, Ayuso C, et al. Dual-Energy CT: Oncologic Applications. Am J Roentgenol. 2012;199(5_ supplement):S98-S105.

14. Baxa J, Matouskova T, Krakorova G, Schmidt B, Flohr T, Sedlmair M, et al. Dual-phase dual-energy $C T$ in patients treated with Erlotinib for advanced non-small cell lung Cancer: possible benefits of iodine quantification in response assessment. Eur Radiol. 2016;26(8):2828-36.

15. Park YN, Hg K, Chon CY, Park JB, Sohn JH, Yang SH, et al. Histological grading and staging of chronic hepatitis standardized guideline proposed by the Korean study Group for the Pathology of digestive diseases. J Pathol Transl Med. 1999;33(5):337-46.

16. Chou C-T, Chen R-C, Lin W-C, Ko C-J, Chen C-B, Chen Y-L. Prediction of microvascular invasion of hepatocellular carcinoma: preoperative $\mathrm{CT}$ and Histopathologic correlation. Am J Roentgenol. 2014;203(3):W253-W9.

17. Chou C-T, Chen R-C, Lee C-W, Ko C-J, Wu H-K, Chen Y-L. Prediction of microvascular invasion of hepatocellular carcinoma by pre-operative CT imaging. Br J Radiol. 2012;85(1014):778-83.

18. Yang CB, Zhang S, Jia YJ, Yu Y, Duan HF, Zhang XR, et al. Dual energy spectral $C T$ imaging for the evaluation of small hepatocellular carcinoma microvascular invasion. Eur J Radiol. 2017:95:222-7.

19. An C, Kim DW, Park YN, Chung YE, Rhee H, Kim MJ. Single hepatocellular carcinoma: preoperative MR imaging to predict early recurrence after curative resection. Radiology. 2015;276(2):433-43.
20. Matsui O, Kobayashi S, Sanada J, Kouda W, Ryu Y, Kozaka K, et al. Hepatocelluar nodules in liver cirrhosis: hemodynamic evaluation (angiography-assisted CT) with special reference to multi-step hepatocarcinogenesis. Abdom Imaging. 2011;36(3):264-72.

21. Nishie A, Yoshimitsu K, Asayama Y, Irie H, Tajima T, Hirakawa M, et al. Radiologic detectability of minute portal venous invasion in hepatocellular carcinoma. Am J Roentgenol. 2008;190(1):81-7.

22. Xu X, Zhang H-L, Liu Q-P, Sun S-W, Zhang J, Zhu F-P, et al. Radiomic analysis of contrast-enhanced $\mathrm{CT}$ predicts microvascular invasion and outcome in hepatocellular carcinoma. J Hepatol. 2019;70(6):1133-44.

23. Lv $P$, Lin XZ, Li J, Li W, Chen K. Differentiation of small hepatic Hemangioma from small hepatocellular carcinoma: recently introduced spectral CT method. Radiology. 2011;259(3):720-9.

24. Richter S, Mücke I, Menger MD, Vollmar B. Impact of intrinsic blood flow regulation in cirrhosis: maintenance of hepatic arterial buffer response. Am J Physiol Gastrointest Liver Physiol. 2000;279(2):G454-62.

25. Gülberg $\vee$, Haag K, Rössle M, Gerbes AL. Hepatic arterial buffer response in patients with advanced cirrhosis. Hepatology. 2002;35(3):630-4.

26. Van Beers BE, Leconte I, Materne R, Smith AM, Jamart J, Horsmans $Y$. Hepatic perfusion parameters in chronic liver disease: dynamic CT measurements correlated with disease severity. AJR Am J Roentgenol. 2001; 176(3):667-73.

27. Hermoye L, Laamari-Azjal I, Cao Z, Annet L, Lerut J, Dawant BM, et al. Liver segmentation in living liver transplant donors: comparison of semiautomatic and manual methods. Radiology. 2005;234(1):171-8.

28. Rios Velazquez E, Aerts HJWL, Gu Y, Goldgof DB, De Ruysscher D, Dekker A, et al. A semiautomatic CT-based ensemble segmentation of lung tumors: comparison with oncologists' delineations and with the surgical specimen. Radiother Oncol. 2012;105(2):167-73.

29. Scholten ET, Jacobs C, van Ginneken B, van Riel S, Vliegenthart R, Oudkerk $M$, et al. Detection and quantification of the solid component in pulmonary subsolid nodules by semiautomatic segmentation. Eur Radiol. 2015;25(2): 488-96.

30. Heye T, Merkle EM, Reiner CS, Davenport MS, Horvath JJ, Feuerlein S, et al. Reproducibility of dynamic contrast-enhanced MR imaging. Part II. Comparison of intra- and Interobserver variability with manual region of interest placement versus semiautomatic lesion segmentation and histogram analysis. Radiology. 2013;266(3):812-21.

\section{Publisher's Note}

Springer Nature remains neutral with regard to jurisdictional claims in published maps and institutional affiliations.

Ready to submit your research? Choose BMC and benefit from:

- fast, convenient online submission

- thorough peer review by experienced researchers in your field

- rapid publication on acceptance

- support for research data, including large and complex data types

- gold Open Access which fosters wider collaboration and increased citations

- maximum visibility for your research: over $100 \mathrm{M}$ website views per year

At $\mathrm{BMC}$, research is always in progress.

Learn more biomedcentral.com/submission 